\title{
Internação hospitalar de pessoas idosas de um grande centro urbano brasileiro e seus fatores associados
}

\author{
Hospitalization of Older People in a Large Brazilian Urban Center and its Associated Factors
}

Renan Lucas da Silva'
Bruno Matida Bonando
Gerson de Souza Santos ${ }^{2}$ (D)
Alessandro Ferrari Jacinto ${ }^{3}$ (D)
Luciano Magalhães Vitorino $^{1}$ (D)

\section{Resumo}

Objetivo: Avaliar a frequência de Internação Hospitalar (IH) nos últimos doze meses em pessoas idosas atendidos na Atenção Primária à Saúde (APS) e seus fatores associados por meio de uma Avaliação Geriátrica Ampla (AGA). Métodos: Estudo transversal, com amostra aleatória de 400 pessoas idosas atendidas em uma Unidade Básica de Saúde (UBS). A avaliação da frequência de IH por pelo menos 24 horas foi autorreferida (sim; não). Utilizou-se questionário sociodemográfico e de saúde, instrumentos para avaliar as atividades básicas e instrumentais da vida diária, status cognitivo, sintomas depressivos, queda e medo de cair. A regressão logística múltipla foi utilizada para investigar os fatores associados à IH. Resultados: A média de idade foi de 75,23 ( $\pm 8,53), 63,2 \%$ dos participantes eram do sexo feminino, $62,6 \%$ relataram um estado de saúde ruim/razoável e 38\% relataram hospitalização nos últimos doze meses. Idade mais avançada, com pior percepção de saúde, doenças crônicas, uso diário de medicamentos, dependentes para as atividades básicas e instrumentais da vida diária, comprometimento do status cognitivo e queda no ano anterior demonstraram associação com a hospitalização. Saber ler e escrever foi associado com menor risco de hospitalização. Conclusão: A frequência de IH de pessoas idosas atendidas em UBS foi alta e foi associada a fatores modificáveis e não modificáveis, indicando que a abordagem multidimensional é uma ferramenta importante no cuidado da pessoa idosa na atenção primária à saúde.

\footnotetext{
Faculdade de Medicina de Itajubá (FMIt), Grupo Afya. Itajubá, MG, Brasil.

Centro Universitário Ages, Departamento de Medicina. Irecê, BA, Brasil.

Escola Paulista de Medicina (Unifesp), Programa de Pós-Graduação Medicina Translacional. São Paulo, SP, Brasil.
}

Palavras-chave: Idosos.
Hospitalização. Geriatria.
Avaliação Geriátrica.

Os autores declaram não haver conflito na concepção deste trabalho.

Não houve financiamento para a execução deste trabalho. 


\section{Abstract}

Objective: To evaluate the frequency of Hospital Admission (HA) in the last twelve months in older adults treated at Primary Health Care (PHC) and its associated factors, through a Comprehensive Geriatric Assessment (CGA). Methods: Cross-sectional study, with a random sampling of 400 older adults using PHC. The frequency of HA for at least 24 hours was self-reported (yes; no). A sociodemographic and health survey was used, tools to evaluate basic and instrumental daily life activities, cognition, depression, falling, and fear of falling. The association of factors to $\mathrm{HA}$ was analyzed using multiple logistic regression analysis. Results: Mean age was $75.23( \pm 8,53), 63.2 \%$ of participants were female $62.6 \%$ reported a poor/fair state of health and $38 \%$ reported HA in the previous twelve months. Older patients, with a poor perceived health, chronic illnesses, daily use of medications, dependent for basic and instrumental daily life activities, cognitive impairment, and having fallen in the previous year demonstrated associations with hospitalization. Knowing how to read and write was associated with protection from hospitalization. The frequency of hospitalization was high in this study. Conclusion: The frequency of HA of older people attended at basic health units was high and was associated with modifiable and nonmodifiable factors, indicating that the multidimensional approach is an important tool in the care of the older adults in primary health care settings.

\section{INTRODUÇÃO}

A população idosa utiliza com maior frequência os serviços de saúde, envolvendo maior custo, tratamento prolongado e comprometimento da etapa de recuperação ${ }^{1}$. A internação hospitalar $(\mathrm{IH})$ nessa população é de alto risco devido a presença de multicomorbidades ${ }^{1}$. Na literatura, encontramse informações relevantes dos fatores de riscos à IH na população idosa ${ }^{1,2}$. No entanto, observa-se a necessidade de uma abordagem multidimensional centrada na pessoa idosa, considerando-se fatores modificáveis e não modificáveis ${ }^{2}$.

Devido ao aumento da população idosa haverá maior demanda por atenção à saúde em todos os níveis e, consequentemente, aumento da frequência de $\mathrm{IH}^{3}$. As pessoas idosas utilizam mais os serviços de saúde do que outras faixas etárias ${ }^{3}$. Além disso, o perfil de doença das pessoas idosas exige mais recursos do sistema de saúde, principalmente, a nível de hospitalização ${ }^{3,4}$. A Atenção Primária à Saúde (APS) tem papel fundamental na redução das taxas de IH nessa população ${ }^{3-6}$. $\mathrm{Na}$ maioria das vezes, as pessoas idosas podem evitar o desfecho IH por meio de medidas eficazes da APS $^{3-6}$. Não há dúvidas que há um grande esforço por parte dos geriatras, gerontólogos, gerontologistas e pesquisadores para identificar os fatores de risco à $\mathrm{IH}$ em pessoas idosas $^{3}$. Estudos anteriores mostraram que as doenças crônicas não transmissíveis (DCNTs), especialmente: multimorbidades, incapacidade funcional, percepção de saúde ruim, polifarmácia, baixa escolaridade e idade avançada estavam associados ao risco de $\mathrm{IH}$ em brasileiros mais velhos ${ }^{5-8}$.

Conhecer os fatores associados à $\mathrm{IH}$ de pessoas idosas é fundamental para o desenvolvimento de políticas de prevenção dos danos causados por esse desfecho ${ }^{5}$. Por esse motivo, torna-se imprescindível a realização de avaliação multidimensional focada na pessoa idosa ${ }^{2}$. Nesse sentido, a Avaliação Geriátrica Ampla (AGA) tem se mostrado a forma mais adequada de avaliar as necessidades da pessoa idosa 9 .

Até onde sabemos, poucos estudos utilizaram uma avaliação multidimensional com uso de escalas validadas sobre capacidade funcional, saúde mental, histórico de quedas, medo de quedas e IH entre pessoas idosas brasileiras atendidas em unidades de APS. Portanto, a prevenção da IH nessa população é relevante para preservar a qualidade de vida e reduzir os custos do sistema de saúde ${ }^{5-7}$. Assim, o objetivo deste estudo foi avaliar a frequência de IH nos últimos doze meses em pessoas idosas atendidos na APS e seus fatores associados, por meio de AGA. 


\section{MÉTODOS}

Estudo transversal com amostra probabilística de pessoas com 60 anos ou mais, atendidas em uma das unidades de APS da cidade de São Paulo, SP, Brasil. Este estudo seguiu as recomendações do Strengthening the Reporting of Observational Studies in Epidemiology $(\mathrm{STROBE})^{10}$. A pesquisa foi desenvolvida na Unidade Básica de Saúde (UBS) Marcus Belenzinho (Belenzinho), localizada na zona leste do município de São Paulo, SP. A UBS possui aproximadamente 40.000 indivíduos cadastrados, dos quais 5.000 são pessoas idosas. Quatrocentos indivíduos foram necessários para fazer modelos de regressão logística hierárquica com uma variável dependente e quinze variáveis independentes, frequência média de $14 \%$ \% . IH de pessoas idosas, valor alfa de $5 \%$ de significância $(\alpha=0,05)$ e poder estatístico de $90 \%$. A seleção das pessoas idosas foi feita por meio de amostragem aleatória simples, usando os 5.000 números de registros de pessoas idosas registradas.

Os critérios de inclusão foram ter 60 anos ou mais e estar cadastrado na UBS Belenzinho. Os critérios de exclusão foram ter uma limitação física grave e diagnóstico médico de deficit cognitivos ou demenciais. A coleta de dados foi realizada entre fevereiro e agosto de 2018 por um enfermeiro com doutorado e mais de dez anos de experiência na APS. Após a seleção aleatória dos potenciais participantes da pesquisa, os escolhidos foram contatados durante as consultas de enfermagem na UBS. As entrevistas (com duração de aproximadamente 40 minutos) foram realizadas individualmente em ambiente privado. A frequência de IH foi obtida por meio de pergunta subjetiva e autorreferida: Você ficou internado por mais de 24 horas nos últimos 12 meses (sim, não)? Variáveis sociodemográficas: sexo (masculino, feminino); faixa etária (65 a 70 anos; 70 a 79; 80 ou mais); estado civil (com companheiro, sem companheiro); saber ler/ escrever (sim; não); nível de estudos (nenhum; 1 a 4 anos; >4 anos); morar sozinho (sim; não).

Variáveis relacionadas ao estado de saúde: satisfação com a vida (sim; não), percepção de saúde (ruim; regular; boa; excelente), doença crônica (sim; não), uso de medicamentos (sim; não), polifarmácia - uso diário de $\geq 5$ medicamentos (sim; não); uso de tabaco (sim; não).
Atividades básicas de vida diária: Índice de Katz - criado por Sidney Katz em 1976 e validado para o português em $2008^{11}$. Esse instrumento é utilizado para avaliar as Atividades Básicas de Vida Diária (ABVD), de acordo com o grau de independência nos resultados de seis funções das ABVDs (banho, vestir-se, ir ao banheiro, transferência, continência e alimentação). A pontuação varia de 0 a 6 pontos e classifica os pacientes em independentes (pontuação zero) e dependentes (pontuação maior ou igual a 1) ${ }^{11}$.

Atividades instrumentais de vida diária: Escala de Lawton - elaborada por Lawton e Brody em 1969 , validada para o português em $2008^{12}$. Esse instrumento é utilizado para avaliar as Atividades Instrumentais da Vida Diária (AIVD), de acordo com escores que variam de 0 a 21. Ele classifica os pacientes em dependentes (pontuação menor ou igual a 20) e independentes (pontuação igual a 21) ${ }^{12}$.

Cognição: Mini Exame do Estado Mental (MEEM) - elaborado por Folstein em 1976, utilizado para avaliar a função cognitiva, adaptado transculturalmente para o português brasileiro em $1994^{13}$. A pontuação varia entre um mínimo de 0 e máximo de 30 pontos. Um dos pontos de corte utilizados no Brasil foi baseado no nível de escolaridade (em anos de estudo): 13 pontos para analfabetos; 18 para escolaridade baixa a média (até oito anos de educação formal), 26 para pessoas idosas com escolaridade alta (mais de oito anos de escolaridade $)^{13}$.

Depressão: Escala de Depressão Geriátrica, forma abreviada com 15 itens (GDS-15); desenvolvido pela Yesavage em 1983 e validado para o português em $2005^{14}$. É uma escala de quinze itens, com duas opções de respostas (sim; não). A pontuação varia de 0 a 15 e classifica os pacientes como sem depressão (menor ou igual a 5) e com depressão (igual ou maior que 6$)^{14}$.

Medo de quedas: A Escala de Eficácia de Quedas - Internacional (FES-I) foi desenvolvida por Yardley em $2005^{15}$. Essa escala foi validada para o português em 2010, com alfa de Cronbach igual a 0,96 ${ }^{15}$. A FES-I avalia o medo de cair em 16 atividades diárias distintas. A escala de pontuação da FES-I, de 16 (nada preocupado) a 64 (extremamente preocupado), com cada item medido em uma escala Likert de quatro pontos. Os pontos de corte para medo de cair 
foram os seguintes: $16-22=$ preocupação baixa e 23 $64=$ preocupação alta $^{15}$. As variáveis para presença de quedas foram história de quedas (sim; não) e queda anterior (<12 meses; sim; não).

Presença de comorbidades com Prince et al. ${ }^{16}$, Cardiovascular (sim; não), neoplasia (sim; não), pulmonar (sim; não), musculoesquelética (sim; não), neurológica (sim; não), metabólica (sim; não), obesidade (sim; não) ${ }^{16}$.

A análise descritiva foi apresentada em valores absolutos e relativos (variáveis categorizadas) e medidas de tendência central (variáveis numéricas contínuas). A proporção das variáveis categorizadas foi comparada por meio do teste qui-quadrado (ou teste de Fisher, de acordo com o tamanho da amostra). A Regressão Logística Múltipla e Hierárquica foi utilizada com a variável dicotômica IH (sim, não). Foram desenvolvidos cinco modelos: Modelo 1: sociodemográfico (idade, gênero, leitura / escrita, estado civil, arranjo familiar), Modelo 2: Modelo 1 + estado de saúde (percepção de saúde (ruim), doença crônica, uso de medicamentos diariamente, polifarmácia - $\geq 5$ medicamentos por dia, uso de tabaco. Modelo 3: Modelo $2+$ capacidade funcional (Katz - dependente ou independente, Lawton dependente ou independente). Modelo 4: Modelo 3 + saúde mental (Depressão - GDS15 sim; não, função cognitiva - MEEM). Modelo 5: Modelo $4+$ quedas (quedas nos últimos 12 meses sim; não, medo de queda - FES-I-16 sim; não). Nível de significância de $5 \%$ foi escolhido para o teste, com $95 \%$ de confiança intervalo e $p<0,05 *$; $p<0,01 * * ; p<0,001 * * *$.

Este projeto foi aprovado pelo Comitê de Ética e Pesquisa da Secretaria Municipal de Saúde de São Paulo, Protocolo 2.468.315, de 17 de janeiro de 2018. Todos os participantes assinaram o Termo de Consentimento Livre e Esclarecido. A pesquisa está de acordo com a Resolução no 466/2012.

\section{RESULTADOS}

Das 488 pessoas idosas deste estudo, 400 (83,33\%) responderam todos os questionários. Das que não participaram, 50 pessoas idosas não atenderam aos critérios de inclusão e 38 recusaram-se a participar do estudo. As variáveis sociodemográficas e de saúde encontram-se na Tabela 1. A média de idade foi de $75,23( \pm 8,53)$ anos, sendo $63,20 \%$ eram do sexo feminino, $67 \%$ não tinham companheiro, 39,5\% não sabiam ler e escrever e $31,5 \%$ moravam sozinhos. Mais da metade (54,2\%) das pessoas idosas não estavam satisfeitas com a vida. Quanto à autopercepção do estado de saúde, 62,6\% avaliaram sua saúde como ruim ou regular e 92,3\% apresentaram DCNT.

$\mathrm{Na}$ Tabela 2, encontramos a prevalência das principais doenças crônicas não transmissíveis.

A frequência de internação nos últimos doze meses foi de 38,0\% (IC 95\%=33,30\%-42,70\%). A regressão logística múltipla não ajustada evidenciou que pessoas idosas, com idade mais avançada, percepção de saúde regular ou péssima, portadoras de doenças crônicas, uso diário de medicamentos, dependentes de ABVD e AIVD e queda nos últimos doze meses foram associadas a maior risco de internação. As variáveis saber ler e escrever e ter melhor estado cognitivo estiveram associadas a menor risco de hospitalização (Tabela 3).

$\mathrm{Na}$ Tabela 4, foram realizados cinco modelos de Regressão Logística Hierárquica. No primeiro modelo, "Variáveis Sociodemográficas", saber ler e escrever apresentou menor risco de ser hospitalizado $(\mathrm{OR}=0,45$; IC95\%: 0,29 - 0,68, $p<0,001)$. No $2^{\circ}$ modelo "Variáveis Sociodemográficas e Estado de Saúde", pessoas idosas com autopercepção negativa do estado de saúde (OR: 10,65; IC95\%: 1,19-95,23, $p=0,034)$ e tabagistas apresentaram maiores chances de $\mathrm{IH}$. Hospitalizado $(\mathrm{OR}=3,41$; IC 95\% $=1,44-8,07, p=0,005)$. No terceiro modelo, "Variáveis Sociodemográficas, Estado de Saúde e Capacidade Funcional", as pessoas idosas dependentes de ABVD-Katz apresentaram maior risco de $\mathrm{IH}(\mathrm{OR}=2,93$; IC95\%=1,42-6,01, $p=0,003)$. No último modelo, as pessoas idosas com percepção de saúde ruim $(\mathrm{OR}=13,49$; IC95\%=1,26-144,38, $p=0,031)$ ou percepção de saúde razoável $(\mathrm{OR}=11,82$; IC95\%=1,12-123,86, $p=0,039)$, usuários de tabaco $(\mathrm{OR}=3,36$; IC $95 \%=1,36-8,29, p=0,008)$, dependentes de ABVD-Katz (OR=2,39; IC95\%=1,18-4,87, $p=0,016)$ e queda nos doze anteriores mês $(\mathrm{OR}=2,37$; IC95\% $=1,09-5,15, p=0,028)$ apresentaram maiores chances de IH. 
Tabela 1. Caracterização sociodemográfica e de saúde das pessoas idosas (n=400). São Paulo,SP, 2018.

\begin{tabular}{|c|c|}
\hline Variáveis & $\mathrm{n}(\%)$ \\
\hline \multicolumn{2}{|l|}{ Idade (anos) } \\
\hline $60-69$ & $104(26,00)$ \\
\hline $70-79$ & $159(39,70)$ \\
\hline$\geq 80$ & $137(34,30)$ \\
\hline \multicolumn{2}{|l|}{ Sexo } \\
\hline Masculino & $147(36,80)$ \\
\hline Feminino & $253(63,20)$ \\
\hline \multicolumn{2}{|l|}{ Estado conjugal } \\
\hline Com companheiro & $132(33,00)$ \\
\hline Sem companheiro & $268(67,00)$ \\
\hline \multicolumn{2}{|l|}{ Sabe Ler/Escrever } \\
\hline Sim & $242(60,50)$ \\
\hline Não & $158(39,50)$ \\
\hline \multicolumn{2}{|l|}{ Anos de estudo } \\
\hline Nenhum & $158(39,50)$ \\
\hline 1 a 4 & $242(60,50)$ \\
\hline$>4$ & $0(0)$ \\
\hline \multicolumn{2}{|l|}{ Mora sozinho } \\
\hline Sim & $126(31,50)$ \\
\hline Não & $274(68,50)$ \\
\hline \multicolumn{2}{|l|}{ Satisfação com a vida } \\
\hline Sim & $183(45,80)$ \\
\hline Não & $217(54,20)$ \\
\hline \multicolumn{2}{|l|}{ Percepção de saúde } \\
\hline Péssima & $89(22,30)$ \\
\hline Regular & $161(40,30)$ \\
\hline Boa & $120(30,00)$ \\
\hline Ótima & $30(7,40)$ \\
\hline \multicolumn{2}{|l|}{ Doença crônica } \\
\hline $\operatorname{Sim}$ & $369(92,30)$ \\
\hline Não & $31(7,70)$ \\
\hline \multicolumn{2}{|c|}{ Uso de medicamentos $^{*}$} \\
\hline Sim & $363(90,70)$ \\
\hline Não & $37(9,30)$ \\
\hline \multicolumn{2}{|l|}{ Polifarmácia } \\
\hline Sim & $148(37,00)$ \\
\hline Não & $252(63,00)$ \\
\hline \multicolumn{2}{|l|}{ Tabagismo } \\
\hline $\operatorname{Sim}$ & $110(27,50)$ \\
\hline Não & $290(72,50)$ \\
\hline \multicolumn{2}{|l|}{ Medo de cair ${ }^{* *}$} \\
\hline $\operatorname{Sim}$ & $251(62,70)$ \\
\hline Não & $149(37,30)$ \\
\hline \multicolumn{2}{|l|}{ Última queda } \\
\hline$<12$ meses & $51(20,30)$ \\
\hline Não & $200(79,70)$ \\
\hline
\end{tabular}

*diariamente; ${ }^{* *}$ Ponto de corte da escala FES-I para medo de cair 16-22= não e 23-64= $\operatorname{sim}^{15}$ 
Tabela 2. Prevalência de doenças crônicas não transmissíveis (n=400). São Paulo,SP, 2018.

\begin{tabular}{ll}
\hline Comorbidades* & $\mathrm{n}(\%)$ \\
\hline Cardiovascular & $295(73,70)$ \\
Sim & $105(26,30)$ \\
Não & $19(4,70)$ \\
\hline Neoplasia & $381(95,30)$ \\
Sim & \\
Não & $26(6,50)$ \\
\hline Pulmonar & $374(93,50)$ \\
Sim & $118(29,50)$ \\
Não & $282(70,50)$ \\
\hline Musculoesquelético & \\
Sim & $199(49,80)$ \\
Não & $201(50,20)$ \\
\hline Neurológico & \\
Sim & $206(51,50)$ \\
Não & $194(48,50)$ \\
\hline Cardiometabólico & \\
Sim &
\end{tabular}

*As doenças crônicas foram categorizadas de acordo com Prince et al. ${ }^{22}$

Tabela 3. Fatores associados à internação hospitalar das pessoas idosas (n=400). São Paulo, SP, 2018.

\begin{tabular}{|c|c|c|}
\hline \multirow{2}{*}{ Variáveis sociodemográficas e clínicas } & Internação ${ }^{a}$ & \multirow{2}{*}{ Valor- $p$} \\
\hline & OR não ajustado (IC95\%) & \\
\hline Idade (média) & $1,09(1,06-1,12)$ & $<0,001 * * *$ \\
\hline Sexo (feminino) & $1,08(0,71-1,65)$ & 0,691 \\
\hline Ler/escrever (sim) & $0,45(0,29-0,68)$ & $<0,001 * * *$ \\
\hline Estado conjugal (sim) & $1,41(0,91-2,19)$ & 0,117 \\
\hline Arranjo Familiar (sim) & $0,91(0,58-1,41)$ & 0,677 \\
\hline Percepção de saúde (péssima) & $5,62(2,09-15,11)$ & $<0,001 * * *$ \\
\hline Percepção de saúde (regular) & $2,92(1,13-7,54)$ & $0,026^{*}$ \\
\hline Percepção de saúde (boa) & $1,10(0,40-2,99)$ & 0,842 \\
\hline Doença crônica (sim) & $3,44(1,29-9,17)$ & $0,013^{*}$ \\
\hline Uso de Medicamentos (sim) & $2,38(1,06-5,36)$ & $0,036^{*}$ \\
\hline Polifarmácia (sim) & $1,35(0,89-2,05)$ & 0,150 \\
\hline Tabagismo (sim) & $0,90(0,57-1,43)$ & 0,678 \\
\hline Katz Total (média) & $6,31(3,88-10,26)$ & $<0,001 * * *$ \\
\hline Lawton Total & $4,29(2,78-6,60)$ & $<0,001 * * *$ \\
\hline Depressão (sim) & $0,77(0,50-1,01)$ & 0,222 \\
\hline MEEM (média) & $0,83(0,76-0,90)$ & $<0,001 * * *$ \\
\hline FES-I (sim) & $1,74(0,81-3,71)$ & 0,150 \\
\hline Última Queda (sim) & $2,93(1,51-5,69)$ & $0,001 * * *$ \\
\hline
\end{tabular}

${ }^{a}$ Internação de pelo menos 24 horas nos últimos 12 meses= 0=não; 1=sim; OR: Odds Ratio; IC: Intervalo de Confiança; MEEM: Mini Exame do Estado Mental; FES-I: Escala de Eficácia de Quedas - Internacional $p<0,05^{*} ; p<0,01^{* *} ; p<0,001^{* * *}$ 
Tabela 4. Regressão logística hierárquica entre variáveis independentes e risco de internação (n=400). São Paulo,SP, 2018.

\begin{tabular}{|c|c|c|}
\hline \multirow{2}{*}{ Modelos } & \multicolumn{2}{|l|}{ Internação ${ }^{a}$} \\
\hline & OR ajustado (IC95\%) & Valor- $p$ \\
\hline \multicolumn{3}{|l|}{$1^{\circ}$ Modelo } \\
\hline Idade (média) & $1,07(0,99-1,15)$ & 0,056 \\
\hline Sexo (feminino) & $0,72(0,39-1,32)$ & 0,295 \\
\hline Ler/escrever (Sim) & $0,45(0,29-0,68)$ & $<0,001 * * *$ \\
\hline Estado conjugal (Sim) & $1,79(0,91-3,49)$ & 0,087 \\
\hline Arranjo Familiar (Sim) & $0,77(0,41-1,42)$ & 0,405 \\
\hline \multicolumn{3}{|l|}{$2^{\circ}$ Modelo } \\
\hline Percepção de saúde (péssima) & $10,65(1,19-95,23)$ & $0,034^{*}$ \\
\hline Doença crônica (sim) & $1,08(0,12-9,37)$ & 0,939 \\
\hline Uso de Medicamentos (sim) & $2,75(0,55-13,62)$ & 0,214 \\
\hline Polifarmácia (sim) & $1,21(0,67-2,20)$ & 0,511 \\
\hline Tabagismo (sim) & $3,41(1,44-8,07)$ & $0,005^{* *}$ \\
\hline \multicolumn{3}{|l|}{$3^{\circ}$ Modelo } \\
\hline Katz Total (dependente) & $2,93(1,42-6,01)$ & $0,003^{* *}$ \\
\hline Lawton Total (dependente) & $1,91(0,97-3,76)$ & 0,061 \\
\hline \multicolumn{3}{|l|}{$4^{\circ}$ Modelo } \\
\hline Depressão (sim) & $1,06(0,55-2,02)$ & 0,852 \\
\hline MEEM (média) & $0,92(0,80-1,06)$ & 0,270 \\
\hline \multicolumn{3}{|l|}{$5^{\circ}$ Modelo } \\
\hline Idade (média) & $1,04(0,99-1,09)$ & 0,087 \\
\hline Sexo (feminino) & $0,07(0,41-1,84)$ & 0,724 \\
\hline Ler/escrever (sim) & $1,44(0,70-2,94)$ & 0,316 \\
\hline Estado conjugal (sim) & $1,25(0,62-2,51)$ & 0,526 \\
\hline Percepção de saúde (péssima) & $13,49(1,26-144,38)$ & $0,031 *$ \\
\hline Percepção de saúde (regular) & $11,82(1,12-123,86)$ & $0,039 *$ \\
\hline Percepção de saúde (boa) & $5,54(0,51-59,54)$ & 0,157 \\
\hline Doença crônica (sim) & $1,07(0,10-10,64)$ & 0,952 \\
\hline Uso de Medicamentos (sim) & $3,29(0,61-17,64)$ & 0,164 \\
\hline Polifarmácia (sim) & $1,19(0,64-2,21)$ & 0,563 \\
\hline Tabagismo (sim) & $3,36(1,36-8,29)$ & $0,008^{* *}$ \\
\hline KATZ Total (dependente) & $2,39(1,18-4,87)$ & $0,016^{*}$ \\
\hline Lawton Total (dependente) & $1,72(0,89-3,34)$ & 0,106 \\
\hline Depressão (sim) & $1,10(0,58-2,08)$ & 0,759 \\
\hline MEEM (média) & $0,92(0,80-1,06)$ & 0,286 \\
\hline FES-I (sim) & $0,92(0,20-4,16)$ & 0,914 \\
\hline Última Queda (sim) & $2,37(1,09-5,15)$ & $0,028^{*}$ \\
\hline
\end{tabular}

${ }^{a}$ Internação pelo menos por 24 horas nos últimos 12 meses= 0=não; 1=sim; KATZ: Atividades Básicas de Vida Diária; LAWTON: Atividades Instrumentais de Vida Diária; MEEM: Mini Exame do Estado Mental; FES-I: Escala de Eficácia de Quedas - Internacional. OR: Odds Ratio; IC: Intervalo de Confiança. ${ }^{*} p<0,05^{*} ; p<0,01 * * ; p<0,001 * * *$. 


\section{DISCUSSÃO}

Este estudo pesquisou a frequência de $\mathrm{IH}$ e os fatores associados em pessoas idosas atendidas na atenção primária. A frequência de IH na amostra estudada foi maior do que em outras publicações brasileiras $^{5-7}$. Pessoas idosas com idade mais avançada, percepção de saúde ruim, portadores de doenças crônicas, uso diário de medicamentos, dependência de ABVD, AIVD e queda nos últimos doze meses apresentaram maiores chances de IH. No entanto, pessoas mais velhas que sabiam ler/escrever e com melhor estado cognitivo demonstraram menos chances de IH.

Esses achados são consistentes com a literatura científica e são importantes para profissionais de saúde que atendem pessoas idosas desde a atenção primária à terciária ${ }^{3-8}$. Nesse contexto, a identificação de indicadores associados a maiores chances de hospitalização representa uma ferramenta essencial na prática clínica, corroborando com medidas preventivas e resolutivas ${ }^{3-8}$. A prevalência de internação na amostra estudada foi maior quando comparada a outras publicações nacionais que variaram entre $7,6 \%$ e $17,7 \% 0^{5-7}$. Possíveis explicações para a maior fragilidade da amostra decorrem de que essas pessoas idosas eram atendidas em uma unidade de APS, quase um terço tem mais de 80 anos, a grande maioria deles tem pelo menos uma DCNT e faz uso de medicação diariamente, a maioria tem percepção de ruim saúde ou insatisfação com a vida e histórico de quedas.

Pessoas idosas com percepções de problemas de saúde apresentam maiores chances de IH. A autopercepção negativa da saúde é compartilhada por diversos estudos como um importante indicador de saúde ${ }^{5,17-19}$. Um estudo de base populacional com 23.815 pessoas idosas evidenciou que a percepção de saúde precária tinham 1,35 mais chance de serem hospitalizados 5 . A avaliação da autopercepção de saúde é uma importante ferramenta subjetiva na prática clínica, de fácil aplicação e excelente triagem para desfechos de saúde, atuando como preditor de hospitalização e morte em populações idosas ${ }^{5,20}$.

O uso de tabaco esteve associado à IH na amostra estudada. Em estudo prospectivo populacional com seguimento de 7,2 anos e 188.167 indivíduos com média de idade de 55 anos, o tabagismo foi associado à IH e mortalidade por diversas doenças cardiovasculares ${ }^{21}$. Dados da literatura destacam o efeito deletério do uso do tabaco, incluindo redução da expectativa de vida ${ }^{21,22}$. Em estudo nacional, o uso de tabaco pelas mulheres subtraiu 4,47 anos de vida em relação às que não usavam e, na população masculina, o impacto foi de 5,03 anos para fumantes ${ }^{22}$. Além disso, o uso do tabaco compromete a qualidade de vida, devido às morbidades a ele relacionadas, como doenças cardiovasculares, doença pulmonar obstrutiva crônica (DPOC) e câncer ${ }^{21}$. Em outro estudo internacional, a fragilidade de pessoas idosas foi maior em fumantes, principalmente na faixa de 60 a $79 \operatorname{anos}^{23}$.

Pessoas idosas dependentes de ABVD apresentaram maior chance de IH. Diversos estudos têm mostrado que a Capacidade Funcional (CF) é uma composição dinâmica que se manifesta como elemento central da saúde da população idosa ${ }^{5-7,24}$. O comprometimento de atividades como tomar banho, alimentar-se e vestir-se está relacionado ao aumento da fragilidade em pessoas idosas e, consequentemente, à maior demanda por atendimento médico e ao risco de $\mathrm{IH}^{5,24}$.

O comprometimento da CF é mais evidente e traz implicações para a vida do idoso, pois infringe sua autonomia, ocasionando uma pior avaliação de sua qualidade de vida e, consequentemente, maior necessidade de intervenção médica e hospitalização ${ }^{5}$. E, além disso, como a literatura tem mostrado, o aumento das hospitalizações pode resultar da deterioração das ABVD do idoso $0^{5-7,24}$.

Uma história de quedas nos últimos doze meses está relacionada a maiores taxas de IH. Entre $1996 \mathrm{e}$ 2012, ocorreram cerca de 66.876 mortes por quedas no Brasil e 941.923 hospitalizações em pessoas com 60 anos ou mais ${ }^{25}$. De acordo com o Center for Disease Control and Prevention (CDC), as quedas são a principal causa de morbimortalidade em pessoas idosas nos Estados Unidos da América (EUA) ${ }^{26}$. Em 2014, quase $28,7 \%$ de pessoas idosas nos EUA relataram queda, resultando em 29 milhões de quedas, 37,5\% das quais necessitaram de $\mathrm{IH}^{27}$. Na população idosa existe estreita relação com o histórico de quedas como indicador de fragilidade e morbidade grave ${ }^{27,28}$. 
Os participantes do estudo que sabiam ler/escrever apresentaram menor propensão à IH. O maior nível de alfabetização é fator de proteção à piores desfechos de saúde ${ }^{29}$, em especial entre a população idosa ${ }^{30}$. Por outro lado, há fortes evidências que a baixa alfabetização é fator de risco para desenvolvimento de DCNTs, baixa adesão aos tratamentos e maior mortalidade ${ }^{30}$. Os participantes que tinham melhor status cognitivo também apresentaram menor risco de $\mathrm{IH}^{31}$. Há sólidas evidências que pessoas idosas com melhor status cognitivo apresentam melhores desfechos de saúde ${ }^{31,32}$.

O último modelo expressa fatores de risco modificáveis e não modificáveis relevantes para a hospitalização de pessoas idosas. A utilização de uma abordagem multidimensional pode melhorar a permanência e vinculação à APS. Há fortes evidências que o uso de AGA pelos profissionais de saúde melhora os desfechos de saúde, qualidade de vida, acelera a reabilitação e diminui o risco de IH na população idosa ${ }^{33,34}$. Por outro lado, o cuidado focado na doença gera maior risco de $\mathrm{IH}$, custos e índices de reinternação $0^{3-8}$.

Este estudo possui algumas limitações que devem ser abordadas. O desenho transversal limita a avaliação das relações de causa e efeito. Maior proporção de participantes do sexo feminino limita a avaliação da exposição a homens mais velhos. Além disso, algumas variáveis independentes sofrem influências contextuais, como o estado emocional e físico em que o indivíduo se encontra no momento de ir para a UBS. No entanto, deve-se destacar o uso de uma abordagem multidimensional, contemplando variáveis sociodemográficas, estado de saúde, $\mathrm{CF}$, saúde mental e quedas. Outro ponto importante é a participação de uma população específica de pessoas idosas atendidas em uma UBS. Esses resultados podem ser uma ferramenta importante para os profissionais de saúde que cuidam de pessoas idosas na APS. Recomendamos a realização de estudos longitudinais com amostras maiores em diferentes locais, a fim de identificar possíveis preditores de IH em pessoas idosas.

\section{CONCLUSÃO}

Este estudo identificou fatores associados à IH de pessoas idosas na APS por meio de uma abordagem multidimensional. Pessoas idosas com idade mais avançada, doenças crônicas não transmissíveis, uso diário de medicamentos, história de quedas no último ano, má percepção de saúde, uso de tabaco e incapacidade básica e instrumental para as atividades diárias apresentam maiores chances de internação nos últimos doze meses. Fatores como saber ler e escrever e ter melhor estado cognitivo apresentaram menores chances de ter sido hospitalizado. O conhecimento dos fatores modificáveis e não modificáveis mostra-se um instrumento valioso para o cuidado dessa população que apresenta necessidades específicas.

Editado por: Maria Luiza Diniz de Sousa Lopes

\section{REFERÊNCIAS}

1. Dias RD, Barros JV. Burden of hospitalisation among older people in the Brazilian public health system: a big data analysis from 2009 to 2015. J Epidemiol Community Health. 2019;73(6):537-43.

2. Ebrahimi Z, Patel H, Wijk H, Ekman I, OlayaContreras P. A systematic review on implementation of person-centered care interventions for older people in out-of-hospital settings. Geriatr Nurs. 2021;42(1):213-24.

3. Huntley A, Lasserson D, Wye L, Morris R, Checkland $\mathrm{K}$, England H, et al. Which features of primary care affect unscheduled secondary care use?: a systematic review. BMJ Open. 2014;4(5):e004746.
4. Jorgensen MG, Rodrigo-Domingo M, Andersen S, Riis J, Jakobsen DB, Lerche M, et al. A cohort study of the effects of multidisciplinary in-patient primary care in older adults. Eur Geriatr Med. 2020;11(4):677-84.

5. Bordin D, Cabral LPA, Fadel CB, Santos CBd, Grden CRB. Factors associated with the hospitalization of the elderly: a national study. Rev Bras Geriatr Gerontol. 2018;21(4):439-46.

6. de Melo-Silva AM, Mambrini JVM, de Souza Jr. PRB, de Andrade FB, Lima-Costa MF. Hospitalizations among older adults: results from ELSI-Brazil. Rev Saúde Pública. 2018;52:1-10. 
7. Nunes BP, Soares MU, Wachs LS, Volz PM, Saes MO, Duro SMS, et al. Hospitalization in older adults: association with multimorbidity, primary health care and private health plan. Rev Saúde Pública. 2017;51:43.

8. Wang HHX, Wang JJ, Lawson KD, Wong SYS, Wong MCS, Li FJ, et al. Relationships of Multimorbidity and Income With Hospital Admissions in 3 Health Care Systems. Ann Fam Med. 2015;13(2):164-7.

9. Parker SG, McCue P, Phelps K, McCleod A, Arora S, Nockels K, et al. What is Comprehensive Geriatric Assessment (CGA)?: an umbrella review. Age Ageing. 2018;47(1):149-55.

10. Vandenbroucke JP, von Elm E, Altman DG, Gotzsche PC, Mulrow CD, Pocock SJ, et al. Strengthening the Reporting of Observational Studies in Epidemiology (STROBE): explanation and elaboration. Int J Surg. 2014;12(12):1500-24.

11. Lino VTS, Pereira SRM, Camacho LAB, Ribeiro Filho ST, Buksman S. Cross-cultural adaptation of the Independence in Activities of Daily Living Index (Katz Index). Cad saúde Pública. 2008;24:103-12.

12. Santos RL, Virtuoso Jr. JS. Reliability of the Brazilian version of the Scale of Instrumental Activities of Daily Living. Rev Bras Promoç Saúde. 2008;21(4):290-6.

13. Bertolucci PH, Brucki S, Campacci SR, Juliano Y. The Mini-Mental State Examination in an outpatient population: influence of literacy. Arq Neuropsiquiatr. 1994;52(1):1-7.

14. Paradela EMP, Lourenço RA, Veras RP. Validation of geriatric depression scale in a general outpatient clinic. Rev Saúde Pública. 2005;39(6):918-23.

15. Camargos FF, Dias RC, Dias J, Freire MT. Cross-cultural adaptation and evaluation of the psychometric properties of the Falls Efficacy Scale-International among elderly brazilians (FES-IBRAZIL). Braz J Phys Ther. 2010;14(3):237-43.

16. Prince MJ, Wu F, Guo Y, Robledo LMG, O’Donnell $\mathrm{M}$, Sullivan R, et al. The burden of disease in older people and implications for health policy and practice. Lancet. 2015;385(9967):549-62.

17. Malta DC, Bernal RTI, Lima MG, de Araújo SSC, da Silva MMA, Freitas MIF, et al. Noncommunicable diseases and the use of health services: analysis of the National. Health Survey in Brazil. Rev Saúde Pública. 2017;51:1-10.

18. Confortin SC, Giehl MWC, Antes DL, Schneider IJC, D’Orsi E. Positive self-rated health in the elderly: a population-based study in the South of Brazil. Cad Saúde Pública. 2015;31:1049-60.
19. Ni'meh A, Shojaia H, Darwish H, Giacaman R. Factors associated with self-rated health among elderly Palestinian women: an analysis of crosssectional survey data. Lancet. 2017;390:1-10.

20. Linderholm M, Törnvall E, Yngman-Uhlin P, Hjelm K. Self-rated health, lifestyle habits and risk assessment in 75-year-old persons attending preventive clinic visits with a nurse in primary health care: a cross-sectional study. Prim Health Care Res Dev. 2019;20:e88.

21. Banks E, Joshy G, Korda RJ, Stavreski B, Soga $\mathrm{K}$, Egger S, et al. Tobacco smoking and risk of 36 cardiovascular disease subtypes: fatal and non-fatal outcomes in a large prospective Australian study. BMC med. 2019;17(1):1-10.

22. Pinto MT, Pichon-Riviere A, Bardach A. The burden of smoking-related diseases in Brazil: mortality, morbidity and costs. Cad Saúde Pública. 2015;31:1283-97.

23. Chamberlain AM, Sauver JLS, Jacobson DJ, Manemann SM, Fan C, Roger VL, et al. Social and behavioural factors associated with frailty trajectories in a population-based cohort of older adults. BMJ Open. 2016;6(5):e011410.

24. Calero-García MJ, Ortega AR, Navarro E, Calero MD. Relationship between hospitalization and functional and cognitive impairment in hospitalized older adults patients. Aging Ment Health. 2017;21(11):1164-70.

25. Abreu DROM, Novaes ES, de Oliveira RR, Mathias TAF, Marcon SS. Fall-related admission and mortality in older adults in Brazil: trend analysis. Ciênc Saúde Colet. 2018;23:1131-41.

26. Ballesteros MF, Webb K, McClure RJ. A review of CDC's Web-based Injury Statistics Query and Reporting System (WISQARS ${ }^{\mathrm{TM}}$ ): Planning for the future of injury surveillance. J Safety Res. 2017;61:211-5.

27. Force UPST. Interventions to Prevent Falls in Community-Dwelling Older Adults: US Preventive Services Task Force Recommendation Statement. JAMA. 2018;319(16):1696-704.

28. Hallgren J, Aslan AKD. Risk factors for hospital readmission among Swedish older adults. Eur Geriatr Med. 2018;9(5):603-11.

29. Walters R, Leslie SJ, Polson R, Cusack T, Gorely T. Establishing the efficacy of interventions to improve health literacy and health behaviours: a systematic review. BMC Public Health. 2020;20(1):1-10. 
30. Panagioti M, Skevington SM, Hann M, Howells K, Blakemore A, Reeves D, et al. Effect of health literacy on the quality of life of older patients with longterm conditions: a large cohort study in UK general practice. Qual Life Res. 2018;27(5):1257-68.

31. Shah TM, Weinborn M, Verdile G, Sohrabi HR, Martins RN. Enhancing cognitive functioning in healthly older adults: a systematic review of the clinical significance of commercially available computerized cognitive training in preventing cognitive decline. Neuropsychol Rev. 2017;27(1):62-80.
32. Bhome R, Berry AJ, Huntley JD, Howard RJ. Interventions for subjective cognitive decline: systematic review and meta-analysis. BMJ Open. 2018;8(7):e021610.

33. Ellis G, Gardner M, Tsiachristas A, Langhorne P, Burke $\mathrm{O}$, Harwood RH, et al. Comprehensive geriatric assessment for older adults admitted to hospital. Cochrane Database Syst Rev. 2017;9(9):Cd006211.

34. Åhlund K, Bäck M, Öberg B, Ekerstad N. Effects of comprehensive geriatric assessment on physical fitness in an acute medical setting for frail elderly patients. Clin Interv Aging. 2017;12:1929-39. 